\title{
Wirless Communication, Monitoring and Security System Embedded Safety Helmet for Real Time Applications
}

\author{
Shivaraman Ilango \\ Health Care Technology and Innovation Centre, IIT Madras
}

\begin{abstract}
This paper wireless communication monitoring and security system embedded safety helmet aims at bridging the security problems and communication gap faced by leading research organizations, nuclear power plants, thermal power plants and related industries in times of any hazardous situation, diagnostic works and security breach that will result in the loss of man power and resources. This is a portable instrument with all the features inbuilt in a safety helmet and can eliminate the above mentioned problems building a safe and a secure environment in the near future.
\end{abstract}

Keywords: Camera, Helmet, NFID, Radio frequency, wireless communication

\section{Introduction}

This paper present the details of methodology pursued in implementing a wireless communication monitoring and security system embedded safety helmet. Our main objective and focus is to design an instrument that can be used in hazardous as well highly protected confidential environment that face the problem of security breach and lack of versatile communication during different situations. The design has three phases

1) Authentication

2) Monitoring section for tracking down the person's movement

3) Versatile wireless full duplex communication.

Authentication is through a principle called NFID (near field communication) that is highly advanced and modified version of

RFID technology. This technology is embedded within the helmet.

The monitoring and video tracking is through a small Wireless camera embedded in the front part of the helmet for observing the movement of the person working inside a hazardous environment or for security purpose in a protected area. This uses separate software that helps the Wireless camera to be interfaced with the laptop that makes it portable. The advantage of this software is that snapshots can be taken, videos can be recorded and it has effects and overlays for fine tuning and focusing on what is happening inside the confidential area.

Wireless communication is a high profound full duplex technique through a headset connected with a microphone attached to the helmet and a small portable hanging dial pad that can be attached to person depending on his comfort level. The communication signals cannot be tapped or hacked as the signals are encrypted by codes.

\section{Existing Sysytem}

With existing technology communication within an organization is done using intercom. Here the intercom lines are fixed to a particular place in a room, preferably the table of the person using it. This uses long running chords to connect the various users through a telephone. It involves a costly infrastructure outlay and maintenance. These cannot be carried along with the person when he travels out of the room/ area in which the intercom is installed. It is immobile. Further, it cannot be used if there is damage to the connecting chord, which might be for various reasons including due to fire, natural calamity or any other disaster. In some establishments such as the police department or the security agencies communication is done using wireless communication technology using walkie talkies, where the data and voice signals can be transmitted and received by transceiver circuits using RF free band, which achieves only half duplex. Further, the frequency range is limited to less than 2.4 Gega Hertz. This is without the use of GSM and blue tooth devices. Here there is a more flexible frequency range that is possible and makes long distance communication highly effective.

Further, in communication using the above, data and voice signals are transmitted and received by transceiver circuits in RF free band. Here the dialer initiates the communication by pressing a push button which sends an RF signal to the control room, which in turn authorizes the signal to be transmitted to all other walkie talkies connected on that particular frequency, within that area. This is through satellite communication. Further, 
the person wishing to send a voice signal has to continuously press and hold the push button while talking. Once he releases the button, the transmitting circuit is closed and the voice communication is cut off. The drawback of this is that full duplex is not possible, continuous holding of the push button during talking is required apart from requiring the assistance of a satellite.

\section{Methodlogy:}

There are three phases, in designing this model

1) NFID technology using NFC technique (phase 1).

2) Wireless communication (phase 2).

3) WI-FI closed circuit camera (phase 3).

\subsection{NFID using RFID and NFC Principles:}

NFC is a short-range high frequency wireless communication technology that enables the exchange of data between devices over about a 10 meters distance.

NFC is an upgrade of the existing proximity card standard (RFID) that combines the interface of a smartcard and a reader into a single device. Here the NFC technology is combined with RFID technology, thus giving it a new shape and advantage.

Near Field identification (NFID) is the use of a wireless non-contact system that uses radio-frequency electromagnetic fields to transfer data from a tag attached to an object, for the purposes of automatic identification and tracking. Here the active reader would be the authorized person and the passive reader an unauthorized person. The use of simple encoder and decoder circuits and programming using Kiel $\mathrm{c}$ is the basic principle behind the first phase. Two LEDS of different color, one red and another green would act as communication between the authorized and unauthorized person. As the authorized person would come near, the program in the encoder would be decoded and green light begins to glow indicating the person is authorized and similarly vice versa for the unauthorized were the red LED would glow. Here the intensity of the glowing LED is amplified and allowed to power a solenoid valve which motor to open the doors where the authorized person is seeking entry. When an unauthorized Person tries to seek entry then then the alarm connected as a part of the circuit alerts the control room.

\subsection{Wireless communication:}

In this phase wireless data and voice signals are transmitted and received by transceiver circuits in RF free band. In the data communication, the numerical data from the dial pad is transmitted in the form of RF signal to connect the appropriate telephone line in the remote room. After the appropriate telephone is picked the voice communication is activated and the data communication is cut off. For the voice communication, voice signal is transmitted and received by transceiver from one remote area to another remote area. The voice communication is full duplex that gives it in added advantage to communicate continuously just like a normal telephone.

\subsection{WIFI Camera:}

Wireless cameras are basically described as a wireless transmitter carrying a camera signal. The Camera is wired to a wireless transmitter and the signal travels between the camera and the receiver. This works much like radio. The sound you hear on a radio is transmitted wirelessly and you tune to a certain frequency and hear the sound. Wireless cameras have a channel also. The receiver has channels to tune in and then you get the picture. The wireless camera picture is sent by the transmitter the receiver collects this signal and outputs it to your Computer or TV Monitor depending on the receiver type.

In this process we use a wireless camera that has an integrated transmitter and this camera generates frequency that is modulated using FSK technique and is sent to the the receiver station that demodulates it and amplifies the signals that finely tuned using a tuner to get a clear focus.

This camera signals are integrated to the laptop using a separate software and TV tuner card. This card is connected to the laptop for the purpose of generating and matching the frequencies signals so that the receiver and transmitter stay connected and the clear image without distortion can be accurately viewed on laptop or monitor.

\subsection{NEAR FIELD IDENTIFICATION:}

\section{Implementation}

The Near field identification technique combines the principle of both RFID (Radio Frequency Identification) and NFC (Near

Field Communication) for authorized entry in to a sophisticated environment with various levels of 
authentication

This near field identification is implemented by using an encoder(HT12E) Decoder(HT12D), RF transmitter which transmits signals of $315 \mathrm{Mhz}$ frequency and RF receiver receives signals of $315 \mathrm{Mhz}, 434 \mathrm{Mhz}$ and $402.52 \mathrm{Mhz}$ frequency,9v(volt)DC power supply, alarm. The range of transmission and receiving of signals can be varied by changing the frequency of the transmitter and receiving unit.

\subsubsection{NFID TRANSMITTER (CARD):}

The NFID Card has an Encoder (HT12E) in which the personal identification of the employee has been programmed using Kiel c. the encoder is connected to the RF transmitter. a 9v dc power supply acts as an source of power for the NFID. The NFID Card is different for different employees based on the programming done on the encoder.

\subsubsection{NFID DETECTOR:}

The NFID Receiver is fitted to the fixed on the door or valve adjacent to the door to which the employee wearing the transmitter unit is seeking entry. it has an Decoder(HT12D). The Decoder decodes the program from the NFID Card and if the program matches the value will be actuated and the door will be open and if the program is unmatched the alarm will be blown.

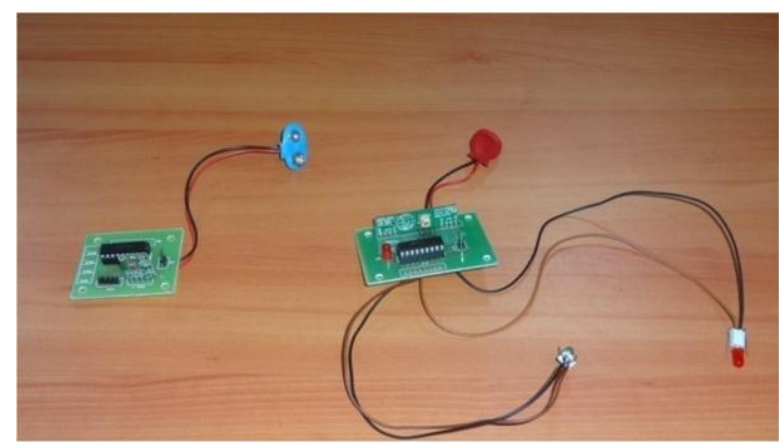

Fig 1

\subsection{WIFI CAMERA:}

This part consists of the Wireless VGA camera that uses a, connected to receiving unit that comprises of power amplifier, frequency tuners, voltage regulator, capacitors, PIC controllers, power supply unit, filters. It also consists of T.V tuner card for interfacing it with the laptop.

The Wireless camera consists of a wireless transmitter connected to a $9 v$ dc power supply and it is fitted to the safety helmet. The transmitting section is also connected to the camera. Video is captured by the camera and transmitted to the receiving unit. The receiving unit is powered by an ac power supply and connected to the monitor or a laptop using interfacing device.

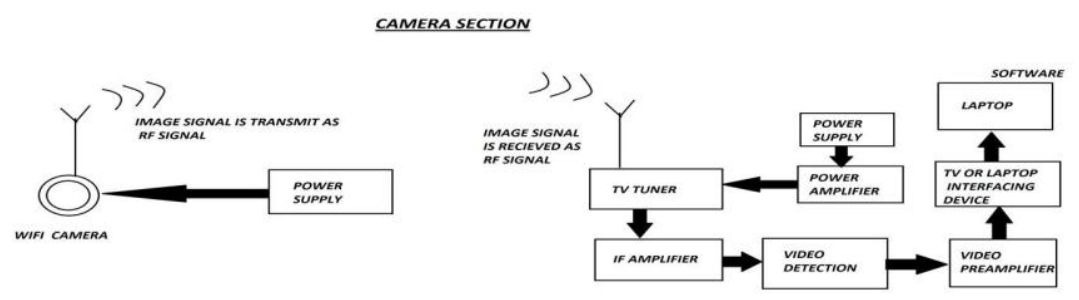

Fig 2.Wireless camera circuit

\subsection{Wireless Communication:}

This is a full duplex communication like a mobile or a chord-less phone. The communication part comprises of two separate units, one to handle voice communication and other to handle data communication, both integrated to function together. The voice communication part comprises of a headset with mike, connected to a pre amplifier, which is in turn connected to a transceiver fitted with an antenna, all of which are connected to a DC power source. This unit also comprises two intercoms which are connected to the base station which acts a link between the voice communication unit and data communication unit. 
The voice communication unit consists of a transceiver, mike pre amplifiers, speaker pre amplifiers and a dc power source. Transceivers transmits RF voice signals in free band where voice signal is transmitted from one transceiver to another transceiver within 50-80 meters and amplifier are used for stabilize the voice signal.

Fig 3. Wireless data communication

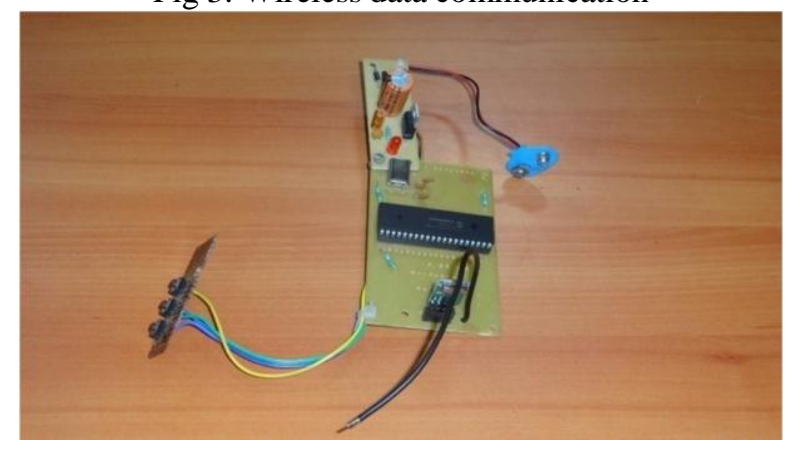

Fig 3. Wireless data communication

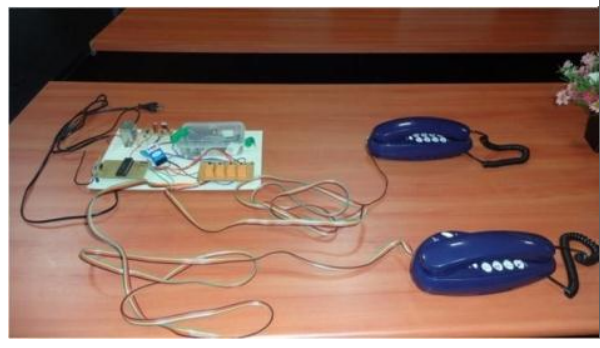

Fig 4. Wireless Voice communication

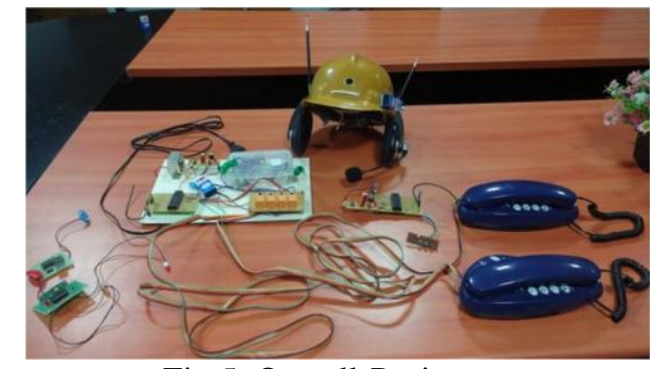

Fig 5. Overall Project

The data communication unit comprises of dial pad which is connected to a PIC microcontroller, which in turn is connected to the base unit through an RF transmitter fitted with a transmitting antenna. The base unit acts a link between the voice communication unit and the data communication unit. It again consists of two parts, one to deal with the receiving of data and other to deal with receiving and transmitting of voice signals. The first part comprises of a receiving antenna to receive the RF signal transmitted by transmitting antennas. These receiving antennas are connected to PIC microcontroller which in turn is connected to the switching relays and the telephone lines. Similarly the voice signals transmitting and receiving antennas are connected through the mic pre amplifier and speaker pre amplifiers. The base station is a portable device and can be plugged in the existing telecom lines patched through the Main distribution frame for communication within the industry.

\section{Conclusion:}

Thus this product as a whole has a wide range of applications in different fields of interest. This technology will bridge the communication gap and security problems faced in many research organizations and will have a positive impact on the society. There is definitely a scope for improvement in this product with development in science and technology.

\section{Future Work:}

We have six wire voice full duplex intercom systems that can be converted in to two wire system using multiplexer. The two wires are DTMF and voice signal together and power supply. These wires are combined to form an integral part and can be inserted in the intercom port for long range communication.

\section{Acknowledgement}

I would like to thank BHAVINI, India's first Prototype fast breeder reactor for providing me an opportunity to work with the renowned scientist and complete this project. This design is a patented and is to be implemented there. 


\section{References}

[1]. Semi-automated CCTV surveillance: The effects of system confidence, system accuracy and task complexity on operator vigilance, reliance and workload N. Dadashi, A.W. Stedmon, T.P. Pridmore

[2]. The impact of CCTV on fundame ntal rights and crime prevention strategies: The case of the Catalan Control Commi ssion of Video surveillance Devices Jose' R. Agustina, Gemma Galdon Clavell,

[3]. Towards a new generation of CCTV networks: Erosion of data Protection safeguards? Fanny Coudert

[4]. Semi-automated CCTV surveillance: The effects of system confidence, system accuracy and task complexity on operator vigilance, reliance and workload N. Dadashi , A.W. Stedmon, T.P. Pridmore

[5]. RFID Security Prof. Dr. Heiko Knospe University of Applied Sciences Cologne, Faculty of Information, Media and Electrical Engineering Prof. Dr. Hartmut Pohl University of Applied Sciences Bonn-Rhein-Sieg, Fachbereich Informatik,

[6]. Wireless and Internet communications technologies for monitoring and control Haydn A. Thompson

[7]. Integrated voice and data transmission employing adaptive modulation in wireless networks Rajarshi Mahapatra*, AnindyaSundarDhar,DebasishDatta 